\title{
Sheet Solid Slicing Method for 3D Models
}

\author{
ZENG Feng \\ School of Computer, Jia Ying University, Meizhou 514015, China \\ 28251245@qq.com
}

Keywords: 3D model, path optimization, slicing method, 3D printing

\begin{abstract}
To against the demerits of slicing methods based on STL model in 3D printing, a sheet solid slicing method of 3D models is presented. In this method, a sheet solid which thickness is equal to the layer thickness is using to intersect with 3D model, and the result of the intersection is target sheet solid which upper and lower surface profiles are the $2 \mathrm{D}$ profiles of two neighbor layers at that position. One half of intersection is saved, and the slicing efficiency is increased. To reduce the idle running distance, the scanning path is optimized by utilizing minimum spanning tree algorithm, and the area filling algorithm is proposed to another manufacturing process. The case study results justified the feasibility and stability. The path optimization algorithm is independent from CAD systems.
\end{abstract}

\section{Introduction}

3D printing integrates multiple technologies such as numerical control technique, CAD, computer graphics, material science and technology, high energy beam machining, and so on ${ }^{[1-3]}$. The 3D CAD model was sliced into a series of $2 \mathrm{D}$ sheets, and then, the cross-section data was extracted to generate the machining codes. The slicing of 3D CAD model is the primary link in the 3D printing ${ }^{[4-5]}$. According to the slicing methods, the slicing technology could be classified into two categories. One is based on indirect model such as STL models, and the other is based on direct slicing CAD models.

At present, most slicing methods are based on STL files. The STL model is a grid-level approximation of 3D model by a large number of triangles. There are some demerits in STL model such as normal vector inversion, crack, overlapping and so on ${ }^{[6-9]}$. In most cases, the intention of designer and the geometrical information of 3D model are included in CAD model. The CAD model itself contains the topological information. The slicing quality could be improved by directly CAD model slicing method.

Based on above-mentioned circumstance, a sheet solid slicing method for 3D CAD model is developed. The thickness of sheet solid is equal to layer thickness of 3D printing. By intersecting sheet solid and 3D CAD model, target sheets are used to extract the cross-section data. Number of intersecting times can be reduced by half.

\section{Technique process of slicing}

The sheet solid presented in this paper is a solid of which the thickness is equal to layer thickness of 3D printing, and the 2D boundary is a little larger then the 3D solid. The sheet solid was build according to the fabrication orientation, and then the target sheet is obtained by intersecting sheet solid and 3D CAD model. The target sheet is the current layer of 3D model which will be 3D printed. Extract cross-section data of target sheet top and bottom surface, and then the original sheet solid move up double thickness to carry on next intersecting. Thus, the times of move and intersection reduce by half. The technique process of sheet solid slicing is shown in Fig.1.

\section{3 key algorithm}

Extract the contour line object of target sheet top and bottom surface through API functions. The contour line object was described as cubic polynomial, shown in formula (1). The $K$ is inherent coefficient of each curve which can be obtained by calling API functions. It determines the form and 
position of a certain curve. Chose $r$ from 0 to 1, and a series 3D coordinates of contour line will be obtained.

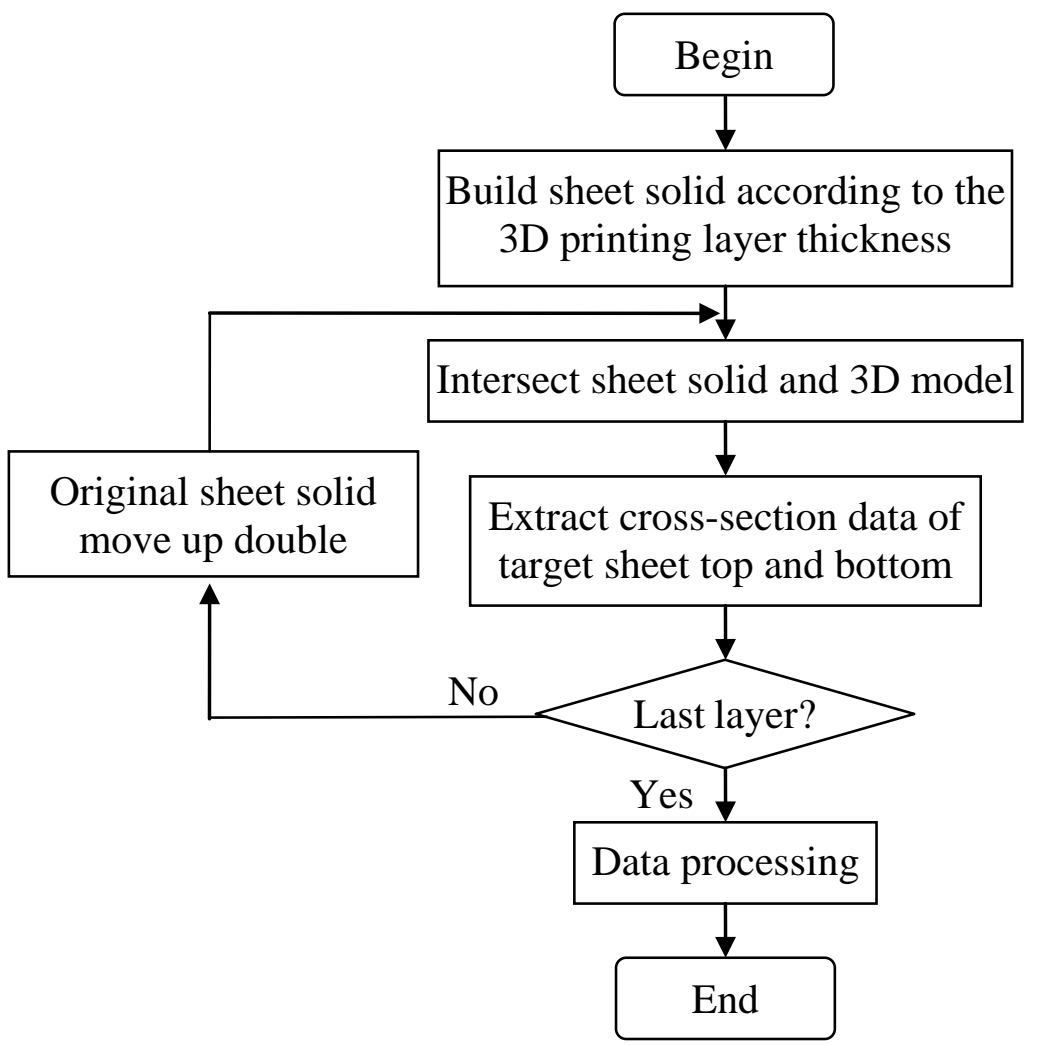

Fig.1 Technique process of sheet solid slicing

$$
\left\{\begin{array}{l}
x=K_{x 0}+K_{x 1} r+K_{x 2} r^{2}+K_{x 3} r^{3} \\
y=K_{y 0}+K_{y 1} r+K_{y 2} r^{2}+K_{y 3} r^{3} \\
z=K_{z 0}+K_{z 1} r+K_{z 2} r^{2}+K_{z 3} r^{3}
\end{array}\right\} \quad(0 \leq r \leq 1)
$$

The CAD coordinate system would be different from the 3D printer's coordinate system. So, the 3D coordinates of contour line obtained by formula (1) must be converted before inputting to the 3D printer. Let $X Y Z$ be the coordinates of CAD system, and $X^{\prime} Y^{\prime} Z^{\prime}$ ' be the coordinates of $3 \mathrm{D}$ printer. The $X$-axis, $Y$-axis and $Z$-axis would be overlapped to the $X^{\prime}$-axis, $Y^{\prime}$-axis and $Z$ '-axis through conversion as follows.

$$
\left[\begin{array}{llll}
X^{\prime} & Y^{\prime} & Z^{\prime} & 1
\end{array}\right]=\left[\begin{array}{llll}
X & Y & Z & 1
\end{array}\right] G_{x}\left(\varphi_{x}\right) G_{y}\left(\varphi_{y}\right) G_{z}\left(\varphi_{z}\right) D\left(D_{x}, D_{y}, D_{z}\right)
$$

In which:

$$
\begin{aligned}
G_{x}\left(\varphi_{x}\right) & =\left[\begin{array}{cccc}
1 & 0 & 0 & 0 \\
0 & \cos \varphi_{x} & \sin \varphi_{x} & 0 \\
0 & -\sin \varphi_{x} & \cos \varphi_{x} & 0 \\
0 & 0 & 0 & 1
\end{array}\right] \\
G_{y}\left(\varphi_{y}\right) & =\left[\begin{array}{cccc}
\cos \varphi_{y} & 0 & -\sin \varphi_{y} & 0 \\
0 & 1 & 0 & 0 \\
\sin \varphi_{y} & 0 & \cos \varphi_{y} & 0 \\
0 & 0 & 0 & 1
\end{array}\right]
\end{aligned}
$$




$$
\begin{gathered}
G_{z}\left(\varphi_{z}\right)=\left[\begin{array}{cccc}
\cos \varphi_{z} & \sin \varphi_{z} & 0 & 0 \\
-\sin \varphi_{z} & \cos \varphi_{z} & 0 & 0 \\
0 & 0 & 1 & 0 \\
0 & 0 & 0 & 1
\end{array}\right] \\
D\left(D_{x}, D_{y}, D_{z}\right)=\left[\begin{array}{cccc}
1 & 0 & 0 & 0 \\
0 & 1 & 0 & 0 \\
0 & 0 & 1 & 0 \\
D_{x} & D_{y} & D_{z} & 1
\end{array}\right]
\end{gathered}
$$

By formula (1), set $r$ from 0 to 1, a series 3D coordinates of contour line will be obtained. And by formula (2), the CAD system coordinates are converted to new coordinates inputted to 3D printer. The lines between the point and point constitute the process path in 3D printing. The 3D printing sample which uses the sheet solid slicing method is shown in Fig.2.

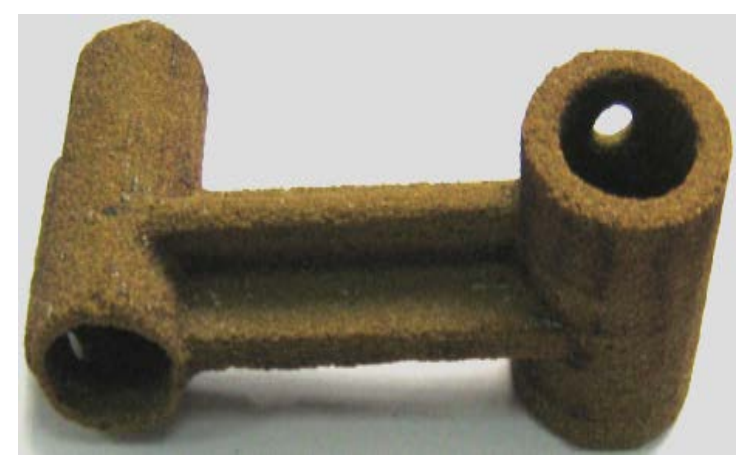

Fig.2 The sample of 3D printing

\section{Summary}

The slicing of 3D CAD model is the primary link in the 3D printing. There are some demerits in STL model based, such as normal vector inversion, crack, overlapping and so on. In this paper, a sheet solid slicing method for 3D CAD model is presented. By intersecting sheet solid and 3D CAD model, target sheets are used to extract the cross-section data. The number of intersecting times can be reduced by half. The practicality and stability of this method were proved by a great number of practical applications. The time-consuming of slicing work can be reduced by this method.

\section{Acknowledgement}

This work was supported by Natural Science Foundation of Guangdong Province \#2014A030307038

\section{References}

[1] Z. J. bin, L. W. Jun. Recent progress in slicing algorithm of rapid prototyping technology. Computer Integrated Manufacturing Systems, Vol. 15, (2009), No. 2, P. 209-221

[2] W. J. Ya, F. Liang, H. J. Bin, Adaptive slicing algorithm of STL model based on feature facets. Application Research of Computers, Vol. 28, (2011), No. 6, P. 2361-2364

[3] A. Wurikaixi, Z. W. Hua, Development of deformation measurement system in direct metal rapid prototyping. Computer Engineering and Applications, Vol. 25, (2008), No. 44, P. 246- 248.

[4] Z. H. Min, C. X. Wen, L. Fen. L. D. Qun, Research on repair algorithms for STL Files. JOURNAL OF COMPUTER-AIDED DESIGN \& COMPUTER GRAPHICS, Vol, 17, (2005), No. 4, P. 761-767 
[5] W. W. Xin, W. Y. Jiang, Z. F. Jun, Applications of CAD/CAE integration based on the interface of STL. OURNAL OF COMPUTER-AIDED DESIGN \& COMPUTER GRAPHICS, Vol, 17, (2005), No. 8, P. 1451-1467

[6] Z. Z. Yu, D. Y. Cheng, H. Jun, A triangulating algorithm for cutting cross-section of STL model. JOURNAL OF COMPUTER-AIDED DESIGN \& COMPUTER GRAPHICS, Vol, 17, (2005), No. 6, P. 1161-1177

[7] R. C. Luo, J. H. Tou, Implementation of a new adaptive slicing algorithm for the rapid prototyping manufacturing system. IEEE/ASME Transactions on Mechatronics, Vol. 9, (2004), No.3, P. 593-600.

[8] G.W., M.Y., Direct slicing from AutoCAD solid models for rapid prototyping. International Journal of Advanced Manufacture Technology, Vol. 21, (2003), No. 10-11 P. 739-742.

[9] S.H.Lo, W.X.Wang, A fast robust algorithm for the intersection of triangulated surfaces. Engineering with computers, Vol. 20, (2004), P. 11-21. 